\title{
Research on Humanoid Robot Soccer System Based on Fuzzy Logic
}

\author{
SHI Lei \\ Xihua University, Chengdu, China \\ Email: shilei19851018@163.com \\ WANG Qiang and Wu lijun \\ Xihua University, Chengdu, China \\ Email:Wq_py123@163.com
}

\begin{abstract}
This paper presents the use of a fuzzy logic controller combined with Machine Vision to improve the accuracy to identify the ball and speed of approaching the ball in Robot Soccer. The conventional robot control consists of methods for path generation and path following. When a robot moves away the desired track, it must return immediately, and while doing so, the obstacle avoidance behavior and the effectiveness of such a path are not guaranteed. So, motion control is a difficult task, especially in real time and high speed control. To achieve good control performance and requirements for timeliness, accuracy to identify and approach the ball, the paper designs a sugeno fuzzy control system, which could make robot complete the whole football performance, including finding, approaching and shooting ball. At the end of the paper, to validate the proposed fuzzy algorithm, the experiment on METALFIGHTER-2 humanoid robot has been run, which showed that the fuzzy controller is effectiveness and robustness.
\end{abstract}

Index Terms - fuzzy system; humanoid robot soccer system; image processing; robot vision.

\section{INTRODUCTION}

Robot Soccer is a high-tech combat activity, and it is the result of combining high-tech and football games people loved .It has been developed rapidly in recent years. Its research areas include robotics, intelligent control, wireless communications, image processing, mechanics and other disciplines and technologies. Through 10 years of development, now the international community has three major events FIRA, RoboCup and RoboCON[1]. Hundreds of teams from dozens of countries participate in the competition each year. However, humanoid robot is the most spectacular game in all competitions. As real-time and dynamic surrounding environment of football game, so it provides a very valuable experimental platform for the research of artificial intelligence theory and other related fields. It's no doubt that fully autonomous robot [2-4] is the mainstream of development in the future following the robot car and semi-autonomous robot .In the context, this article designed an efficient fully autonomous humanoid soccer robot fuzzy control system.
The concept of fuzzy subset has been established in 1965 by L.A. Zadeh[5].Fuzzy control is a practical alternative for a variety of challenging control applications since it provides a convenient method for constructing nonlinear controllers via the use of heuristic information[6]. which means that fuzzy control provides a formal methodology for representing, manipulating, and implementing a human's heuristic knowledge about how to control a system.

Fuzzy logics are many-valued logics that form a suitable basis for logical systems reasoning under uncertainty or vagueness. There are many uncertainties in the real world. Fuzzy theory treats a kind of uncertainty called fuzziness, where it shows that the boundary of yes or no is ambiguous and appears in the meaning of words or is included in the subjunctives or recognition of human beings. Fuzzy theory is essential and is applicable to many systems - from consumer products like washing machines or refrigerators to big systems like trains or subways. Recently, fuzzy theory has been a strong tool for combining new theories (called soft computing) such as genetic algorithms or neural networks to get knowledge from real data.

The fuzzy controller block diagram is given in Fig 1, where we show a fuzzy controller embedded in a closedloop control system. The object controlled outputs are denoted by $\mathrm{y}(\mathrm{t})$, its inputs are denoted by $\mathrm{u}(\mathrm{t})$, and the reference input to the fuzzy controller is denoted by $r(t)$.

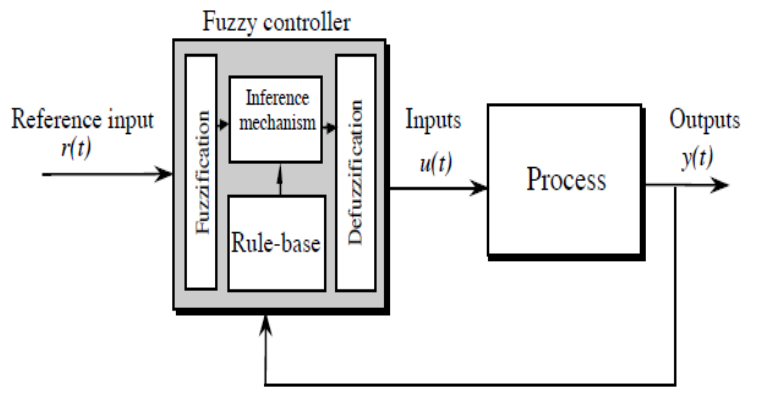

Figure1 Fuzzy Controller architecture.

II. Structure of Humanoid Robot Soccer System 


\section{A Situation on the field}

Fig. 2 shows the situation on the field, Roj is the other side's robot. $\theta_{\mathrm{oj}}$ is the drift angle from the direction of advance of robot $R_{\mathrm{oj}}$ to the ball. $\mathrm{R}_{\mathrm{Hj}}$ is our robot and $\theta_{\mathrm{Hj}}$ is the drift angle from the direct ion of advance of robot $\mathrm{R}_{\mathrm{Hj}}$ to the ball, $\gamma$ is the angle between the ball moving direction and the line joining up ball center point and the opponent's goal line center point.

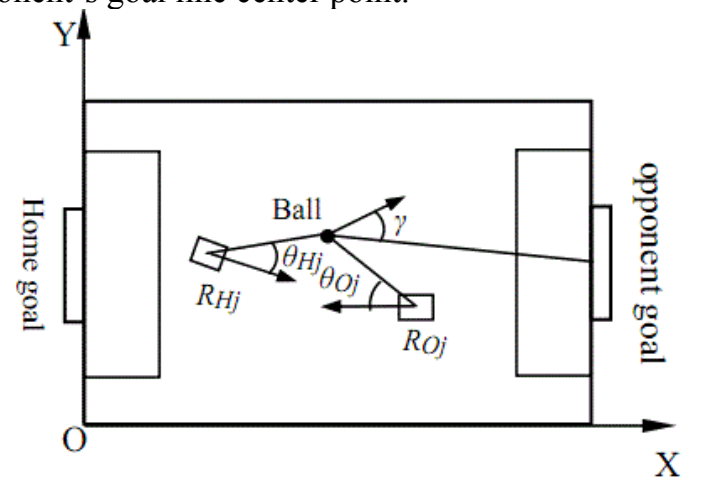

Figure2 situation on the field

In the games, the most common and two basic targets are football and opponent goal. One is using football as the target: First of all, detect the relative position between the soccer and robot via the visual system and then adjust robot posture, finally close to the ball in the best posture; The second is using the enemy's goal as the target: Firstly detect the position of goal, and then adjust the robot posture, finally, robot with ball come near to the other side goal with the best posture [8-9]. Controller is the core part in achieving these goals. This paper is going to use "football" as target to study and design fuzzy logic controller.

At the beginning of the games, Robot should adjust the posture to find the position of ball in the field .secondly combining the direction of the opponent goal, the upper-computer control robot to act, including turning to right, left and so on. finally, robot walk forward directly to the ball and shoot to the goal. Next, it will tell you the details.

\section{$B$ basic construction of humanoid robot soccer system}

The humanoid robot soccer system mainly consists of slave-computer, communication system, and supercomputer. The interaction relationship among them has been shown in Fig 3.

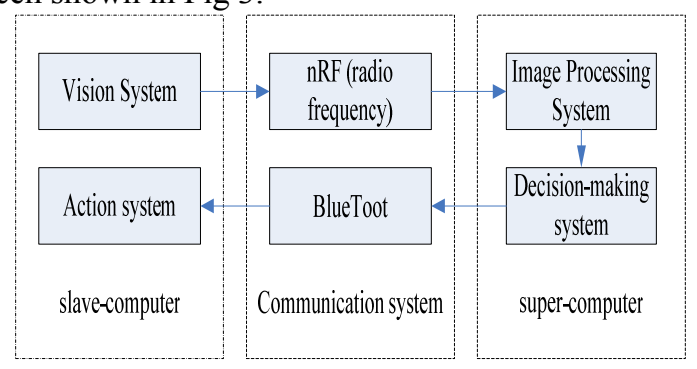

Figure3 .Composition of robot soccer system

Slave-computer mentioned above is robot itself. This system uses METAL-FIGHTER- 2 humanoid robot with camera as a controlled object. The Size of this type of robot is $320 \times 180 \times 90 \mathrm{~mm}$, weight $1.2 \mathrm{~kg}$. It has 17 degrees of freedom (DOF) and it can independently play football and complete many other complex movements. Fig4 displays the full-scale mockup established in PROE3.0. Wireless communication system is mainly used to transfer the action instructions from computer to robot and images data. Motor commands are transmitted by 2.4GHZ spike and robot could use 2400 2500MHZ wireless radio frequency to transfer image data. The super-computer's program is written by Microsoft Visual Studio 2008. The role of super-computer is mainly to realize the imagery processing and design of the decision system and so on.

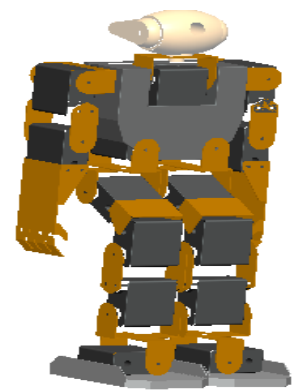

Figure4 .METAL FIGHTER-2 robot model

\section{DESIGNING RoBOT’s BASIC MOVEMENTS}

In order to control the robot to move, control data is too large if motor control instruction is sent directly by the computer. Considering the real-time requirements of robot soccer, this article designed a 27-based low-level action just as table1 shown. When the action code is directly written into robot chip, the decision-making system's task is just sending the corresponding action instruction into robot, which greatly reduces the amount of communication data. In the design of slave-computer action, the system uses robBASICv2.72 as a code editor and METAL FIGHTER-2 robot as a action debugger.

TABLE I. THE NAME OF BASIC ACTION AND THE CORRESPONDING INSTRUCTIONS NUMBER

\begin{tabular}{|c|c|c|}
\hline Right30\&Up70(1) & Right15\&Up70(2) & Walk\&Up70(3) \\
\hline Left15\&Down40(4) & Left70\&Up70(5) & Right30\&Up60(6) \\
\hline Right15\&Up60(7) & Right30\&Down40(8) & Left15\&Up60(9) \\
\hline Left30\&Up60(10) & Right30\&Keep(11) & Right15\&Keep(12) \\
\hline Left30\&Down50(13) & Right15\&Down50(14 & Left30\&Keep(15) \\
\hline Right30\&Down50(16) & Left15\&Keep(17) & Walk\&Down50(18) \\
\hline Left15\&Down50(19) & Walk \& Keep(20) & Walk\&Up60(21) \\
\hline Right15\&Down40(22) & Walk\&Down40(23) & Left15\&Up70(24) \\
\hline Left30\&Down40(25) & LeftShoot(26) & RightShoot(27) \\
\hline
\end{tabular}

Explain: Right 30 -Rotate Right $30^{\circ}$; Left30- Rotate Left $30^{\circ}$; Up70head turn up to $70^{\circ}$; Down50-head turn down to $50^{\circ}$; Walk- Straight walk forward; LeftShoot- Left foot shooting; RightShoot- Right foot shooting; Keep-head keep up with the angle of the previous state; the number (1)-(27) stand for corresponding action instructions

\section{DESIGN OF FuZZY DECISION CONTROL System}


A fuzzy system is a static nonlinear mapping between its inputs and outputs (i.e., it is not a dynamic system)[7]. It is assumed that the fuzzy system has inputs $\mathrm{u}_{i} \in U_{i}$ where $\mathrm{i}=1,2, \ldots, \mathrm{n}$ and outputs $\mathrm{y}_{i} \in Y_{i}$ where $\mathrm{i}=1,2, \ldots, \mathrm{m}$, as shown in Fig5. The inputs and outputs are "crisp"- that is, they are real numbers, not fuzzy sets. The fuzzification block converts the crisp inputs to fuzzy sets, the inference mechanism uses the fuzzy rules in the rule-base to produce fuzzy conclusions (e.g., the implied fuzzy sets), and the defuzzification block converts these fuzzy conclusions into the crisp outputs.

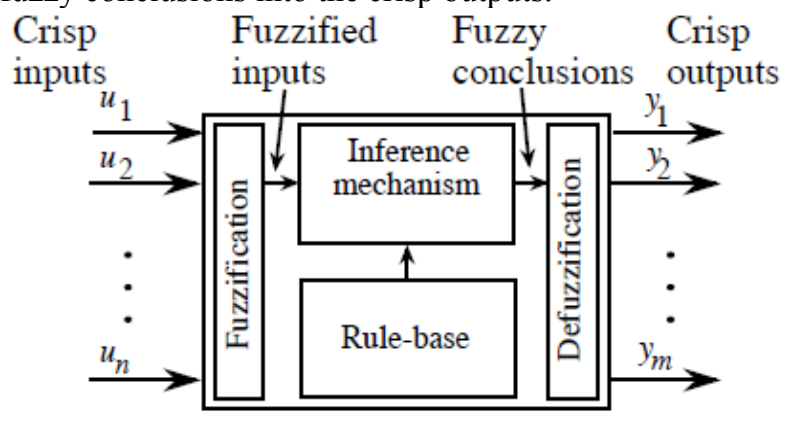

Figure5 Fuzzy System

The whole working process of robot soccer system is shown in Fig7. In the construction of this system, both image processing and fuzzy decision control systems are achieved through programming in the Microsoft visual studio2008 environment. First of all, I'll make several assumptions before designing control system as follows:

1) Monocular camera line of sight is just in front of the robot, that is, an object in front of the robot is in the middle of the images captured by visual.

2) Robot can execute instructions accurately which is sent by PC and walk steadily.

\section{A. Structure and composition of Fuzzy Control system}

Fuzzy control is a form of numerical control, and its composition is similar to the general digital control system. Aimed at Robot Soccer control performance and requirements for timeliness, a Sugeno fuzzy control system is designed in this paper which is used to complete finding, approaching the ball and shooting control.

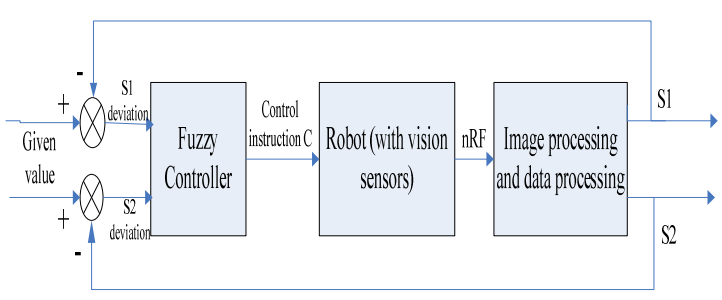

Figure 6. Soccer Robot Modules

Fuzzy Control System of Soccer Robot is mainly composed of fuzzy controller, Robot mechanical systems and processing program of image and other data, which is shown in Fig6 above.

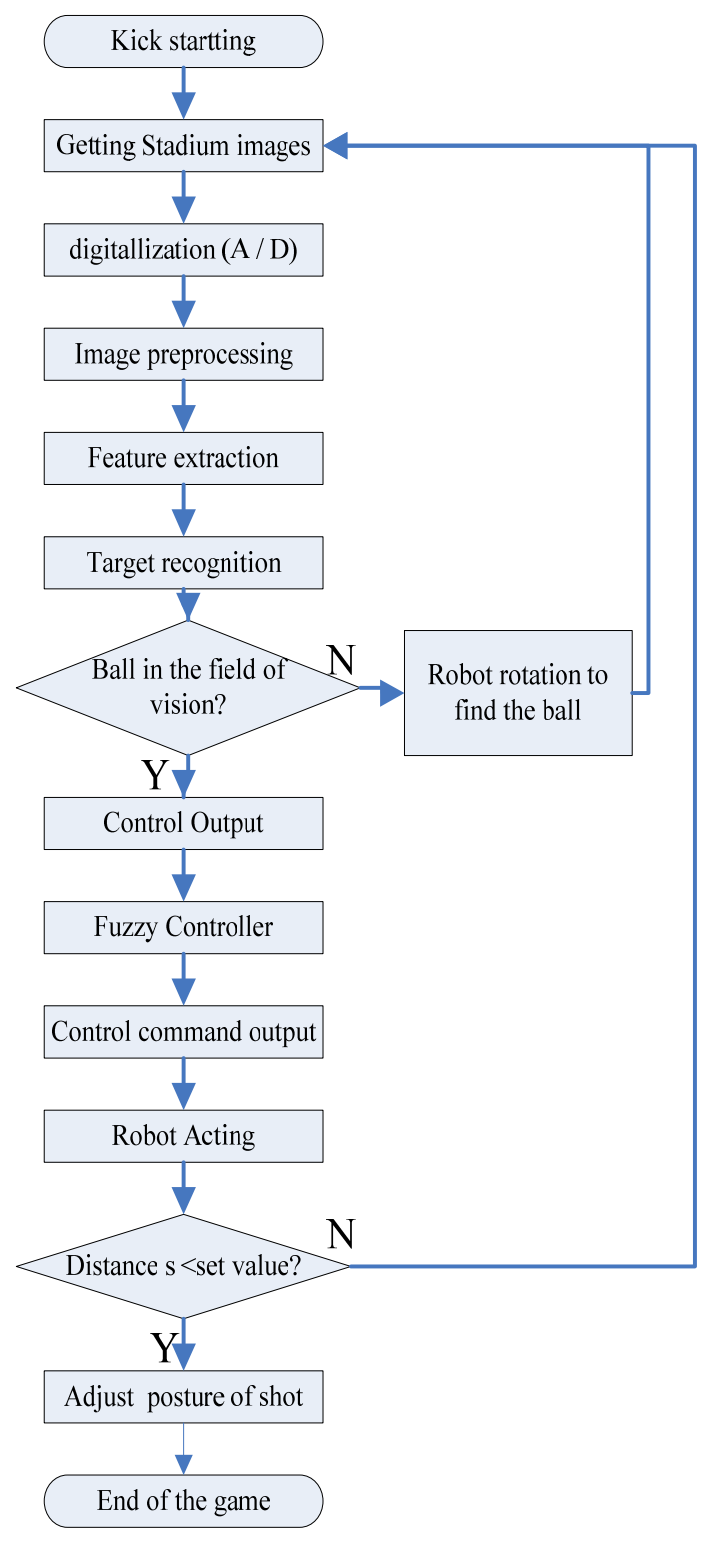

Figure 7 Flow chart of Soccer Robot Working

Fuzzy controller is actually a computer, and it implements a variety of fuzzy algorithms which is necessary in the control process. It is also the "smart" part of the control system. Robot mechanical system is considered as a controlled object which directly receives instructions that are exported by fuzzy controller. Robot vision, image processing and data processing program work together to output the actual ball position value which is used as a feedback value to input. The original image of court which is accessed by the visual is shown in Fig 8-a. By image segmentation, feature extraction and other operations of image processing, we got the following images which are shown in Fig 8-b and c. Then we can get many interesting data through follow-up dataprocessing process. As shown in Fig 8-a, image upper left corner shows the coordinates of the ball's gravity is (101, $69)$ in the Coordinates "O", and the distance between the robot and the ball is $13 \mathrm{~cm}$ and other information. Following, the paper will make a detailed analysis for the design of fuzzy controller. 


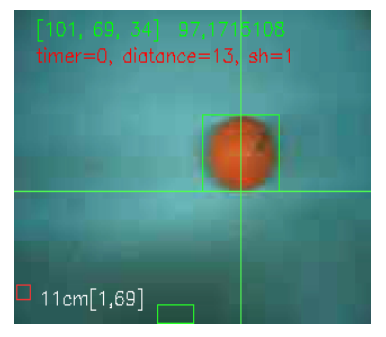

(a) Original Image

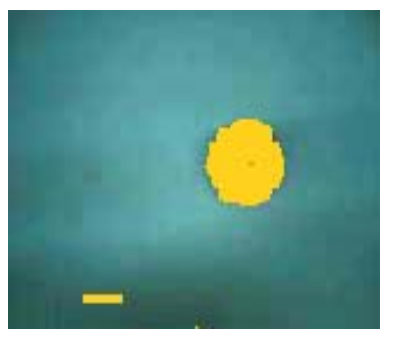

(b) Target Recognition result

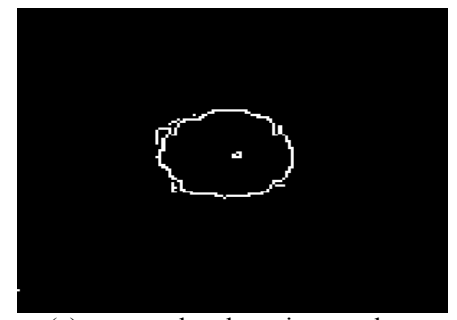

(c) canny edge detection result

Figure8. the Original Image and the Visual Recognition Results

As we know that the main difference between fuzzy control system and usual computer control system is that the fuzzy controller is used. Fuzzy controller is the core of the fuzzy control system. Advantages and disadvantages of a fuzzy system performance depend largely on the structure of fuzzy controller, the use of fuzzy rules, inference algorithms, fuzzy decision synthesis methods and other factors. Following, Allow me to introduce the details step by step.

\section{B. Design of Fuzzy Controller}

Fig9 shows the construction of common Fuzzy logic controller, which has four main components, that is , fuzzification interface for input variables, knowledge base, fuzzy reasoning and defuzzification interface. The fuzzification interface simply modifies the inputs so that they can be interpreted and compared to the rules in the rule-base; The "rule-base" holds the knowledge, in the form of a set of rules, of how best to control the system; The reasoning mechanism evaluates which control rules are relevant at the current time and then decides what the input to the robot should be; the defuzzification interface converts the conclusions reached by the inference

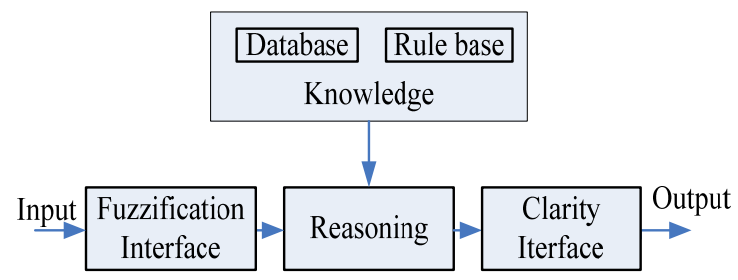

mechanism into the inputs to the robot.

Figure9 Common Fuzzy Controller Construction

The output variables of this control system in the paper are the robot control commands that are constant, so the system adopt Sugeno fuzzy model.

\section{1) choosing the fuzzy controller inputs and outputs}

It must be understood that the choice of the controller inputs and outputs is a fundamentally important part of the control design process. In the paper, it designed a two-input and single-output control system. In order to make robot get near to target quickly, during the design of controller, this paper make the target position deviation that is the difference of expected ball gravity coordinates value and actual output value by data processing system as the system input variables, and here is denoted by $(\Delta \mathrm{s} 1(\mathrm{t}), \Delta \mathrm{s} 2(\mathrm{t}))$. As shown in Fig10, in the robot vision reference frame, S2 and S1 are horizontal and vertical coordinates of football gravity respectively. If both of coordinates S1 and S2 are zero, that is, the ball locate the origin $\mathrm{O} 1$, it indicate that the ball is in the center of robot vision. Otherwise, the fuzzy controller will output corresponding commands to drive robot action.

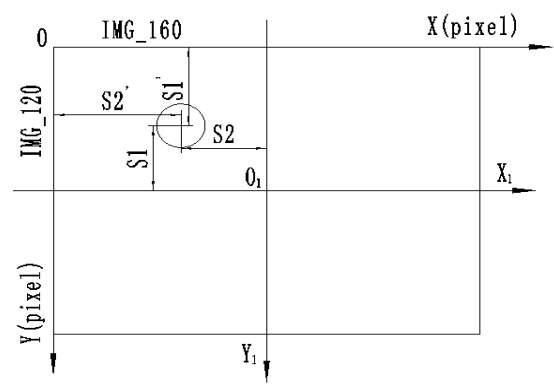

Figure 10. the establishment of variables Coordinate

As mentioned above, so as to avoid to transfer large communication data, the system designed basic moment and saved directly into slave-computer. So supercomputer's function is to send control commend to make robot carry out corresponding action. Thereby, the controller use controlled command number as the output variable. and here is denoted by $\mathrm{C}$.

2) Fuzzy set vocabulary and domain of each variable

The specifications of the fuzzy set vocabulary and variable domain are the foundation of the model. The model's correct behavior as well as its fitness for practical applications depends on well thought-out variable domains. Variable domains are established based on detailed studies of the particular characteristics of the application[10].

Because both of input variables are exact values, to use fuzzy control technology, it's necessary that you should convert them into membership functions of fuzzy sets firstly. In order to facilitate project implementation, use $\mathrm{X}$ and $\mathrm{Y}$ to express the fuzzy subsets of deviation $(\Delta \mathrm{s} 1$, $\Delta \mathrm{s} 2)$. And both of them are divided into 13 grades and expressed as $-6,-5,-4,-3,-2,-1,0,1,2,3,4,5,6$, that is $\mathrm{X}=\mathrm{Y}=\{-6,-5,-4,-3,-2,-1,0,1,2,3,4,5,6\}$. Fuzzy linguistic variables of inputs are denoted by $(\mathrm{NB}, \mathrm{NS}, \mathrm{ZR}$, $\mathrm{PS}, \mathrm{PB})(\mathrm{NB}=$ negative big, $\mathrm{NS}=$ negative small, $\mathrm{ZR}=$ zero, $\mathrm{PS}=$ positive small, $\mathrm{PB}=$ positive big). Each linguistic word set corresponds to a membership function. The output variable is only control command, which is directly used by low-computer, so, the scale 
number of output is 1 . And both of the input subsets are [$24,24]$, so the input scale number is 0.25 . These are listed in the table 2 below.

TABLE II PARAMETER LIST

\begin{tabular}{|c|c|c|c|}
\hline variables & $\Delta s 1$ & $\Delta S 2$ & $\mathrm{C}$ \\
\hline Linguistic variables & $\mathrm{X}$ & $\mathrm{Y}$ & $\mathrm{C}$ \\
\hline $\begin{array}{c}\text { actual bound of } \\
\text { variable }\end{array}$ & \multicolumn{2}{|c|}{$[-24,24]$} & $1,2,3 \cdots 24,25$ \\
\hline Fuzzy subset & $\mathrm{NB}, \mathrm{NS}, \mathrm{ZR}, \mathrm{PS}, \mathrm{PB}$ & \\
\hline $\begin{array}{c}\text { Fuzzy bound of } \\
\text { variable }\end{array}$ & {$[-6,6]$} & $1,2,3 \cdots 24,25$ \\
\hline $\begin{array}{c}\text { Proportional } \\
\text { number }\end{array}$ & $6 / 24=0.25$ & 1 \\
\hline
\end{tabular}

\section{3) Fuzzy control rules and decision making}

The rule-base is constructed so that it represents a human expert "in-the-loop". Hence, the information that we load into the rules in the rule-base may come from an actual human expert who has spent a long time learning how best to control the process. So, control rule is the combination of expert's theoretical knowledge and practical experience. This controller is a double input and single output system, which is constructed just like the type of "IF $\Delta s 1$ and $\Delta S 2$ THEN C". Next, we will use the above linguistic quantification to specify a set of rules (a rule-base) that captures the expert's knowledge about how to control the robot. For the robot controlling problem, with two inputs and five linguistic values for each of these, there are at most $5^{2}=25$ possible rules (all possible combinations of premise linguistic values for two inputs). These fuzzy control rules are shown in Table 3 described below.

Rule 1: If $\Delta s 1=\mathrm{NB}$ and $\Delta s 2=\mathrm{NB}$ then $\mathrm{C}=1$

Rule 2: If $\Delta s 1=\mathrm{NB}$ and $\Delta S 2=\mathrm{NS}$ then $\mathrm{C}=2$

Rule 3: If $\Delta s 1=\mathrm{NB}$ and $\Delta S 2=\mathrm{ZR}$ then $\mathrm{C}=3$

Rule 25: If $\Delta s 1=\mathrm{PB}$ and $\Delta S 2=\mathrm{PB}$ then $\mathrm{C}=25$ such expertise is loaded into the fuzzy controller to automate the reasoning processes and actions of the expert.

TABLE III. TABLE OF FUZZY CONTROL RULE

\begin{tabular}{|c|c|c|c|c|c|c|}
\hline \multicolumn{2}{|c|}{ C } & \multicolumn{5}{|c|}{$\Delta \mathrm{s} 2$} \\
\cline { 3 - 8 } \multicolumn{2}{|c|}{} & $N B$ & $N S$ & $Z R$ & $P S$ & $P B$ \\
\hline \multirow{5}{*}{$\Delta s 1$} & $N B$ & 1 & 2 & 3 & 4 & 5 \\
\cline { 2 - 7 } & $N S$ & 6 & 7 & 8 & 9 & 10 \\
\cline { 2 - 7 } & $Z R$ & 11 & 12 & 13 & 14 & 15 \\
\cline { 2 - 7 } & $P S$ & 16 & 17 & 18 & 19 & 20 \\
\cline { 2 - 8 } & $P B$ & 21 & 22 & 23 & 24 & 25 \\
\hline
\end{tabular}

According to these control rules, we can easily get corresponding control decision $\mathrm{C}$.

4) Foundation of membership function
Membership function of input variables can use triangular, trapezoidal and bell-shaped function and so on. Theoretically, however, Bell is the best. Therefore, this system uses the bell-shaped membership function. Its function expression is given as follows.

$$
f(x, a, b, c)=\frac{1}{1+\left|\frac{x-c}{a}\right|^{2 b}}
$$

In the formula, the parameter $\mathrm{b}$ is usually positive; the parameter $\mathrm{c}$ is used to determine the center of curve.

Through parameter adjustment, ultimately established the input variables $\Delta s 1, \Delta s 2$ membership function which are shown in Fig 11 and Fig 12[10-13].

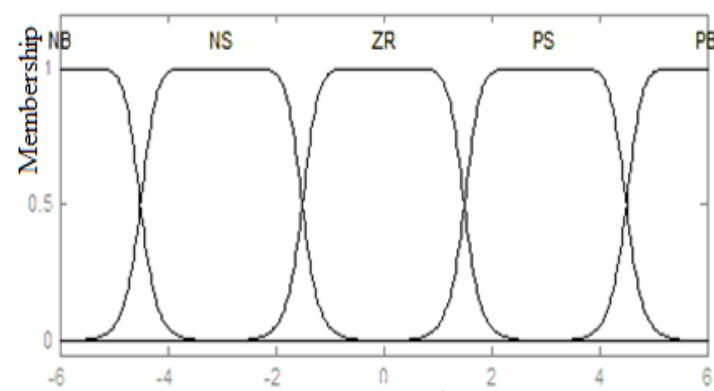

Figure 11 Shape and distribution of $\Delta \mathrm{s} 1$ 's Membership Function

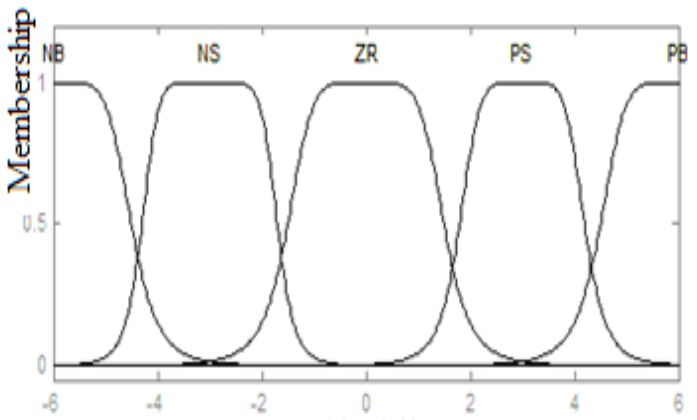

Figure 12 Shape and distribution of $\Delta \mathrm{s} 2$ 's Membership Function

Finally, here established are fuzzy variables table of $\Delta \mathrm{s} 1, \Delta \mathrm{s} 2$ and their membership degree, which has been listed in Table 4 and Table 5.

Table IV. Membership degree of $\Delta s 1$

\begin{tabular}{|c|c|c|c|c|c|c|c|c|c|c|c|c|c|}
\hline \multirow{2}{*}{$\Delta \mathrm{s} 1$} & \multicolumn{13}{|c|}{ u } \\
\hline & -6 & -5 & -4 & -3 & -2 & -1 & 0 & 1 & 2 & 3 & 4 & 5 & 6 \\
\hline $\mathrm{PB}$ & 0 & 0 & 0 & 0 & 0 & [] & 0 & 0 & 0 & 0 & 0.4 & 0.8 & 1 \\
\hline $\mathrm{FS}$ & 0 & 0 & 0 & 0 & 0 & 0 & 0 & 0.3 & 0.7 & 1 & 0.7 & 0.3 & 0 \\
\hline $\mathrm{ZR}$ & 0 & 0 & 0 & 0 & 0 & 0.5 & 1 & 0.5 & 0 & 0 & 0 & 0 & [] \\
\hline NS & 0 & 0.3 & 0.7 & 1 & 0.7 & 0.3 & 0 & 0 & 0 & 0 & 0 & 0 & 0 \\
\hline $\mathrm{HB}$ & 1 & 0.8 & 0.4 & 0 & 0 & 0 & 0 & 0 & 0 & [ & 0 & 0 & 0 \\
\hline
\end{tabular}


Table V. Membership degree of $\Delta S 2$

\begin{tabular}{cccccccccccccc}
\hline & $\Delta s 2$ & \multicolumn{11}{c}{} \\
\cline { 2 - 12 } & -6 & -5 & -4 & -3 & -2 & -1 & 0 & 1 & 2 & 3 & 4 & 5 & 6 \\
\hline PB & 0 & 0 & 0 & 0 & 0 & 0 & 0 & 0 & 0 & 0 & 0.2 & 0.9 & 1 \\
PS & 0 & 0 & 0 & 0 & 0 & 0 & 0 & 0.1 & 0.7 & 1 & 0.7 & 0.1 & 0 \\
ZR & 0 & 0 & 0 & 0 & 0.2 & 0.9 & 1 & 0.9 & 0.2 & 0 & 0 & 0 & 0 \\
NSS & 0 & 0.1 & 0.8 & 1 & 0.8 & 0.1 & 0 & 0 & 0 & 0 & 0 & 0 & 0 \\
NB & 1 & 0.9 & 0.2 & 0 & 0 & 0 & 0 & 0 & 0 & 0 & 0 & 0 & 0 \\
\hline
\end{tabular}

In the paper, Fuzzy decision is Sugeno fuzzy decision, and the solution of fuzzy use average weighted method. The Relationship surface between Fuzzy controller's

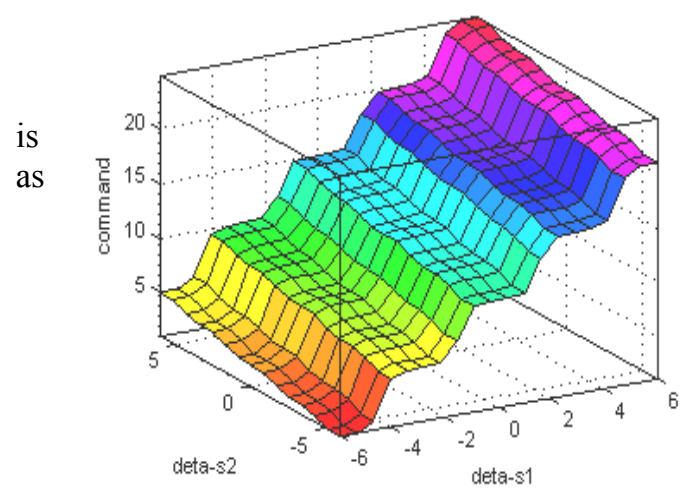

output

and

input

shown

Fig 13

below.

Figure.13. Relationship surfaces between input and output

\section{EXPERIMENTS AND ANALYSIS}

To validate the proposed fuzzy controller, the experiment on METALFIGHTER-2 humanoid robot and simulation in Matlab has been run. In the first experiment, I randomly adjusted the position between the robot and football, and made 10 records of different robot locations that included the values of $(\Delta s 1 \quad \Delta s 2)$ and the corresponding instructions number executed by Robot. What can be drawn easily from the Table 5 below is that the fuzzy logic control system has features of simpleness, high control accuracy and high stability. In particular, compared with the traditional control method, Fuzzy control has a better adaptability for solving the nonlinear system like this.
TABLE VI. EXPERIMENTAL RESULTS

\begin{tabular}{|c|c|c|c|c|c|c|c|}
\hline \multicolumn{2}{|c|}{ Irquut } & \multirow{2}{*}{$\begin{array}{c}\text { Cortollex } \\
\text { Outpunt }\end{array}$} & \multirow{2}{*}{$\begin{array}{l}\text { Eobot } \\
\text { Antion }\end{array}$} & \multicolumn{2}{|c|}{ Irqunt } & \multirow{2}{*}{$\begin{array}{c}\text { Cortwolkx } \\
\text { Outrput }\end{array}$} & \multirow{2}{*}{$\begin{array}{l}\text { Bobot } \\
\text { Antion }\end{array}$} \\
\hline$B=1$ & $\Delta<2$ & & & $\Delta=1$ & $\$ 2$ & & \\
\hline-6 & -6 & 101 & 1 & 0 & 0 & 13 & 13 \\
\hline-56 & -6 & 102 & 1 & 1 & 4 & 14.4 & 14 \\
\hline-6 & -4 & 186 & 2 & 3 & 4 & 19.2 & 19 \\
\hline-3 & -3 & 7 & 7 & 4 & 6 & 20.3 & 20 \\
\hline-2 & -1 & 822 & 8 & 6 & 6 & 25 & 25 \\
\hline
\end{tabular}

\section{CONCLUSION}

Humanoid soccer robot system is a multidisciplinary system, and its application will become increasingly widespread. In this paper, a fuzzy control system structure is completed which is based on visual robot soccer system. The design of basic movements of the lower machine of the system is completed in robobasicv2.72 debugging. Both image processing and fuzzy decision-making control in PC are programmed in Visual Studio 2008. the type of "Sugeno" fuzzy reasoning used in the system has advantages of simple computation and the benefits of mathematical analysis, and it is easy to combine with the methods such as PID control, Optimization and adaptive, thus obtaining with the controller or the fuzzy modeling tools that have the ability of optimization and adaptive.

\section{ACKNOWLEDGMENT}

This work was partly supported by the key Disciplinary of Mechanical Design and Theory of Xihua University (NO.XZD0813-09-1), partly by the Talent introduction and Training project of Xihua University (NO.R0620205), and partly by Key Discipline Construction Project in Sichuan Province (SZD0409-081).

\section{REFERENCES}

[1] HONG Bing-rong,KE Wen-de,YUAN Quan-de.Minihumanoid Robots oriented to Education, Entertainment and Research[J] Journal of Harbin Institute of Technology,2009,41(2):1-5. (in chinese)

[2] LUO Zhen,CAO Qi-xinCHEN Wei-dong. Review On the design of middle size self-determined soccer robot[J],Robot,2003.25(4):384-387

[3] Kim J H, Shim H S ,Jung MJ, et al . Cooperative multi2agentrobotic systems: from the robot2soccer perspective [A]. InProcMIROSOT97 [C], Korea Seoul : Korea Kaist Publisher ,1997.

[4] LIU Yin-ping. Design and Implementation of Soccer Robot Control System[J].Science Technology and Engineering,2009, 14 (7):75-78.

[5] Handbook of Texas Instruments, TMS320F2810 and TMS320F2812 digital signal processors. 2002. 
[6] Fuzzy control / Kevin M. Passino and Stephen Yurkovich. TJ213.P317 1997

[7] P. J. Antsaklis and K. M. Passino. Towards intelligent autonomous control systems: Architecture and fundamental issues. Journal of Intelligent and Robotic Systems, 1:315 - 342, 1989.

[8] Yu Qian. Based on global vision humanoid soccer robot system construction[D].ChengDu:XiHua University,2009 (in Chinese)

[9] Deng meng,LiZu-shu.Humansimulated intelligent control of target tracking for autonomous soccer robot[J] Central South University of Technology(science and technolgy),2007,28(1)

[10] Amaury A. Caballero, Jos é D. Mitrani, Fuzzy Logic methodology for the comparison of costruction firms.

[11] Peng Xiu-yang,Li Yi-dang.Stable walking research of humanoid robot control[J]. Taiyuan University of Technology,2008,3(6) (in Chinese)

[12] Wei Tian Xiu-si.Humanoid Robots[M].Beijing: Tsinghua University Press,2007.3

[13] Li Shi-yong.Fuzzy control ,Neurocontrol and Intelligent Cybernetics[M]. Harbin Institute of Technology Press, 1998.9 (in Chinese)

[14] LouShun-tian,Hu Chan-hua, System analysis and designbased on Matlab-Fuzzy System. Xidian University Press.2001.5 (in Chinese)

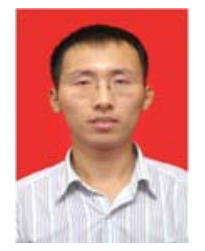

Lei SHI was born in Shanxi, China, in 1985. With good performance, he received his Bachelor's degree in Machine design, manufacturing and automation major in the Department of Mechanical Engineering and Automation from Xihua University, Chengdu, China, in 2009. In the same year, he was recommended for admission to Mechanical Design and Theory of Xihua University with the highest composite score in the department and began to associate in Robotic Researches. During his postgraduate study, he's been to Harbin Institute of Technology quite often to exchange ideas with its relevant personnel about robotic researches. The team led by him won two first places, two second and a third in the "Information Science and Technology
Institute of China Cup," the Tenth National Robotics Competition and the 2010 FIRA Robot World Cup qualifying competition in the Chinese team. His current research interests include Intelligent Robots, Electromechanical Integration Technology, and Modern Control Technology etc.

Qiang WANG, born in Sichuan, in 1964. In 2007, he received the Ph.D. degree in Engineering from Sichuan University. He used to be the director of the Public Basic Teaching Division of the Department in Mechanical Engineering and Automation of Xihua University, and now is the director of Institute of Mechanical Design and Theory, the deputy managing director of Institute of Robots and a member of the southwest branch of Robotic Soccer Association and of the youth branch of Sichuan Mechanical Engineering Association. He worked at Mechanical Research \& Design Institute of Sichuan Institute of Sealing Technology and then at Motorola Electronics Co., Ltd. Asia-Pacific R \& D center in Singapore. He won the title of Chengdu Multi-Skill Teacher and was awarded a first prize and a second in the selection activity of Chengdu's Top Ten University Teachers. He has presided over 1 national natural science foundation project, 3 provincial projects and more than 10 transverse projects. He currently researches on backlash-free double-roller enveloping worm gearing, hybrid institutions and their study of virtual axis machine tool, and fully autonomous multi-robot systems as well as market umbrellas and hanging umbrella automatic transformation. Over 30 of his papers were published in Core journals at home and abroad, and of which 10 were included by SCI, EI, and ISTP.

Lijun WU was born in sichuan, China, in 1989. She will receive her Bachelor's degree in automation major from the Xihua University, Chengdu, China, in 2012.After graduated from xihua university, she plans to work firstly in the field of automation for some years, then studies deeper back to university. 